Original Research

\title{
Lower Extremity Kinematics of the Y-Balance Test in Healthy and ACL Injured Adolescent Females
}

\author{
Alison Bulow, CAT(C), PhD ${ }^{1}$, Alixandra Bellemare, MSc ${ }^{2}$, Judy E Anderson, PhD ${ }^{3}$, Jeff R S Leiter, PhD, Peter B \\ MacDonald, MD, FRCSC ${ }^{4}$, Jason D Peeler, CAT(C), PhD ${ }^{5}$ \\ 1 Department of Human Anatomy \& Cell Science, College of Medicine, University of Manitoba, 2 Faculty of Kinesiology and Recreation Management,
University of Manitoba, ${ }^{3}$ Department of Biological Sciences, University of Manitoba, 4 Department of Surgery, Section of Orthopaedic Surgery,
College of Medicine, University of Manitoba; Pan Am Clinic, 5 Department of Human Anatomy \& Cell Science, College of Medicine, University of
Manitoba; Pan Am Clinic
Keywords: movement system, 2-dimensional video analysis, functional testing, dynamic balance, anterior cruciate ligament
https://doi.org/10.26603/001c.21529
}

International Journal of Sports Physical Therapy

Vol. 16, Issue 2, 2021

\section{Background}

Adolescent females are at significant risk for sustaining an ACL injury. The Y-Balance Test (YBT) is frequently used to evaluate neuromuscular control and lower extremity function. However, few studies have quantified 2D lower extremity kinematics during performance of the YBT, and there is an absence of kinematic data specific to at-risk adolescent females.

\section{Purpose}

To examine lower extremity joint kinematics during execution of the YBT by healthy and ACL-injured adolescent females.

\section{Study Design}

Prospective cohort.

\section{Methods}

Twenty-five healthy and ten ACL-injured (mean time from injury 143 days) adolescent females were assessed using the YBT. Sagittal and frontal plane knee and ankle motion was video recorded during execution of the YBT anterior reach movement. Ankle dorsi-flexion, knee flexion, and knee valgus angles were quantified via kinematic analysis. ANOVAs with a post hoc Bonferroni correction were used to compare YBT scoring (\%LL) and kinematic data between groups. Pearson product-moment correlations determined the relationship between kinematic data and YBT scoring.

\section{Results}

Healthy and ACL-injured subjects demonstrated similar YBT scores and lower extremity kinematic data. Healthy subjects demonstrated a weak positive correlation between ankle dorsiflexion and YBT scoring, and a weak negative correlation between knee valgus and YBT scoring. These relationships did not exist for ACL-injured subjects. Kinematic data for both groups also demonstrated a large degree of variability, regardless of YBT score.

\section{Conclusions}

Adolescent females frequently utilize a variety of lower extremity movement strategies when performing a functional movement task, and scoring on the YBT offers limited insight regarding lower extremity joint kinematics and ACL-injury risk in a physically

\footnotetext{
a Corresponding Author:

Dr. Alison Bulow,

Department of Human Anatomy \& Cell Science,

University of Manitoba,

102-745 Bannatyne Avenue, Winnipeg, Manitoba, R3E 0J9

alison.bulow@gmail.com
} 
active adolescent female population.

\section{Level of Evidence}

Level 3.

\section{INTRODUCTION}

The anterior cruciate ligament (ACL) is one of the most common sites of orthopedic injury, with an annual incidence of 68.6 per 100,000 person-years in the general population. ${ }^{1}$ While risk of ACL injury is significant for both sexes, adolescent females have a 1.6-fold greater rate of ACL injury per athletic exposure than adolescent males, ${ }^{2,3}$ with the peak incidence $(227.6$ per 100,000$)$ of ACL injury occurring in girls $14-16$ years of age. ${ }^{1}$ Research seeking to explain this gender disparity has frequently focused on modifiable biomechanical and neuromuscular factors that may influence lower extremity kinematics and the performance of functional movement tasks. ${ }^{4-8}$

Functional movement testing that involves single-leg squats, lateral step-downs or various jump landing tasks $^{9-13}$ suggests that dynamic knee valgus measures may be predictive of ACL injury. ${ }^{5}$ Initial investigations comparing sexes suggest that females demonstrate greater alterations in lower extremity kinematics, and deficits in dynamic balance and postural stability when performing the same movement tasks. ${ }^{4-8,12}$ Beyond this, the dynamic knee valgus angle has also been demonstrated to influence ankle joint kinematics. ${ }^{13,14}$ As such, further clinical research is needed to clarify the relationship between the knee valgus angle and lower extremity kinematics in an at-risk adolescent female population. $9,10,15$

The Y-Balance Test (YBT) is a dynamic balance test that is frequently used in clinical and research settings to assess lower extremity function. ${ }^{16,17}$ Performance on this test is quantified as a measure of maximal reach distance in a specific direction, or as a calculated composite score (average distance of all reach directions). ${ }^{18}$ Dynamic balance testing is frequently used as a screening tool to identify individuals at risk of lower extremity injury; to assess deficiencies following injury; or to monitor rehabilitation progress. ${ }^{8}$ Lower test scores have previously been associated with an increased risk of ACL injury, ${ }^{19-22}$ ACL-deficiency, ${ }^{8}$ and following ACL reconstruction. ${ }^{23}$ Neuromuscular education and balance training programs have been reported to improve scoring on dynamic balance tests such as the YBT. 6,24 However, few investigators have examined whether a link exists between kinematic joint measures for the lower extremity and performance on dynamic balance tests such as the YBT, ${ }^{23,25-27}$ and currently there is an absence of data which is specific to an at-risk adolescent female population.

The purpose of this investigation was to examine 2D lower extremity joint kinematics during execution of the YBT by healthy and ACL-injured adolescent females. The study had three specific aims: 1) Measure and compare the YBT reach distances for healthy and ACL injured adolescent females; 2) Measure and compare the ankle and knee joint angles of healthy and ACL injured adolescent females when performing the YBT anterior reach movement; and 3) Determine whether a relationship exists between lower extremity kinematic measures and scoring on the YBT ante- rior reach movement in healthy and ACL injured adolescent females.

\section{METHODS}

\section{PARTICIPANTS}

Following institutional ethics approval (H2014:302), healthy and ACL-injured adolescent females were recruited from the community to participate in this clinical study. Twenty-five healthy adolescent females and an exploratory group of 10 ACL-injured adolescent females consented to participate. Healthy subjects reported no recent trauma to the lower extremity; subjects in the ACL-injured group had their injury confirmed via orthopaedic consult and magnetic resonance imaging. All participants completed a standardized screening protocol which indicated that they had no knee joint effusion, were able to fully flex and extend the knee joint through a full range of motion, had no quadriceps lag with an active straight-leg raise, had quadriceps strength of at least $75 \%$ of the unaffected leg and were able to perform 10 consecutive single-legged hops pain free. ${ }^{28}$ All participants were female, 12-18 years of age, with no history of lower extremity injury (other than ACL trauma in the injured group) or concussion in the prior six months. The height, weight, and BMI of all subjects were recorded. Knee joint laxity for all subjects was evaluated using the KT-1000 (MEDmetric Corp., San Diego, CA). ${ }^{29}$ Maturation status was determined using the self-reported pubertal maturation observational scale (PMOS) ${ }^{30}$ leg dominance was determined by preference for kicking a ball; and information regarding sport participation for each participant was documented.

\section{YBT TESTING FOR DYNAMIC BALANCE}

All subjects completed the YBT (Move2Perform, Evansville, IL) according to previously described and standardized procedures. ${ }^{16}$ Following completion of the practice trials, the distance from the YBT apex of the most proximal edge of the reach indicator was recorded for the first test trial while participants performed movements in three directions: anterior (ANT), posteromedial (PM) and posterolateral (PL). The dominant (or ACL deficient) limb served as the support leg. All reach distances were normalized as a percentage of each participant's stance-limb length (\%LL), and measured from the anterior superior iliac spine to the most distal aspect of the ipsilateral medial malleolus in a supine lying position. ${ }^{31}$

\section{KINEMATIC ANALYSIS OF LOWER EXTREMITY JOINT ANGLES}

For the purpose of this investigation, only the YBT anterior (ANT) reach direction was recorded and used for kinematic analysis. Previous research suggests that this reach direction served as a significant discriminator in predicting risk 
of lower extremity injury, ${ }^{19,20,22}$ and pilot testing revealed that kinematic data from the ANT reach direction demonstrated the greatest accuracy. Two HD video cameras (Sony Handycam HDR-UX20, Sony Corp., Minato, Toyko, Japan) were used to collect lower extremity kinematic data while each participant performed the YBT in the ANT direction. One camera was positioned three meters in front of each subject to capture a full frontal-plane view, while the other camera was positioned three meters lateral to each subject to capture a sagittal view. All digital images were coded and saved for subsequent video analysis. Analysis was only completed for the dominant limb of the healthy subjects and the injured limb of the ACL-injured participants. ${ }^{32,33}$

Open-license video analysis software (Kinovea 0.8.15) was then used to quantify three joint angles at the point of maximal ANT reach distance when performing the YBT:

(1) the degree of knee joint flexion in the sagittal plane; (2) the degree of ankle joint dorsiflexion in the sagittal plane; (3) the knee valgus angle in the frontal plane. The same anatomical landmarks were used as reference points for calculating the joint angles of all participants. Selection of these landmarks was based on methodologies previously described in the literature. ${ }^{34-36}$ Each kinematic measure was calculated using the Kinovea software (Figure $1 \mathrm{~A} \& \underline{\mathrm{B}}$ ). The angle of knee flexion in the sagittal plane was determined by measuring the angle created between the lower leg and the posterior thigh with the neutral or starting angle being considered full extension $\left(180^{\circ}\right)$. The angle of ankle dorsiflexion in the sagittal plane was determined by measuring the angle created between the long axis of the foot and the lower leg with neutral being considered $90^{\circ}$ of dorsi-flexion while in a standing position. The angle of knee valgus in the frontal plane was determined by measuring the angle created between the lateral thigh and the lower leg with neutral being considered full extension $\left(180^{\circ}\right)$. This orientation allowed the knee valgus angle to be reported as a positive value.

\section{STATISTICAL ANALYSIS}

A power analysis $\left(n=2\{(1.96+0.84) 6.7 / 8\}^{2}\right)$ from a previous study of healthy recreationally active adolescent females performing the ANT reach of the YBT indicated that a minimum of 12 healthy subjects would be required to adequately power this investigation. ${ }^{36}$ ANOVA testing was used to analyze for differences between the healthy and ACL-injured subjects on demographic, anthropometric, YBT reach distances and the lower extremity joint angles. An alpha level of $p<0.05$ was set to determine statistical significance. The relationship between kinematic angles and the YBT scoring was evaluated using Pearson product-moment correlations. ${ }^{26,37,38}$ Correlation coefficients $(r)$ of $0.25-0.49$, $0.50-0.74$, and $0.75-1.0$ were considered to represent weak, moderate and strong relationships. ${ }^{39}$ The $95 \%$ confidence interval (CI) for the YBT reach distances of the healthy subjects was used to group subjects for comparison. ANOVA testing between groups with a post hoc Bonferroni correction of $p<0.0167$ were used to determine statistical significance. In addition, we performed single-subject analysis for each variable using the healthy subject $95 \%$ CI's for comparison.

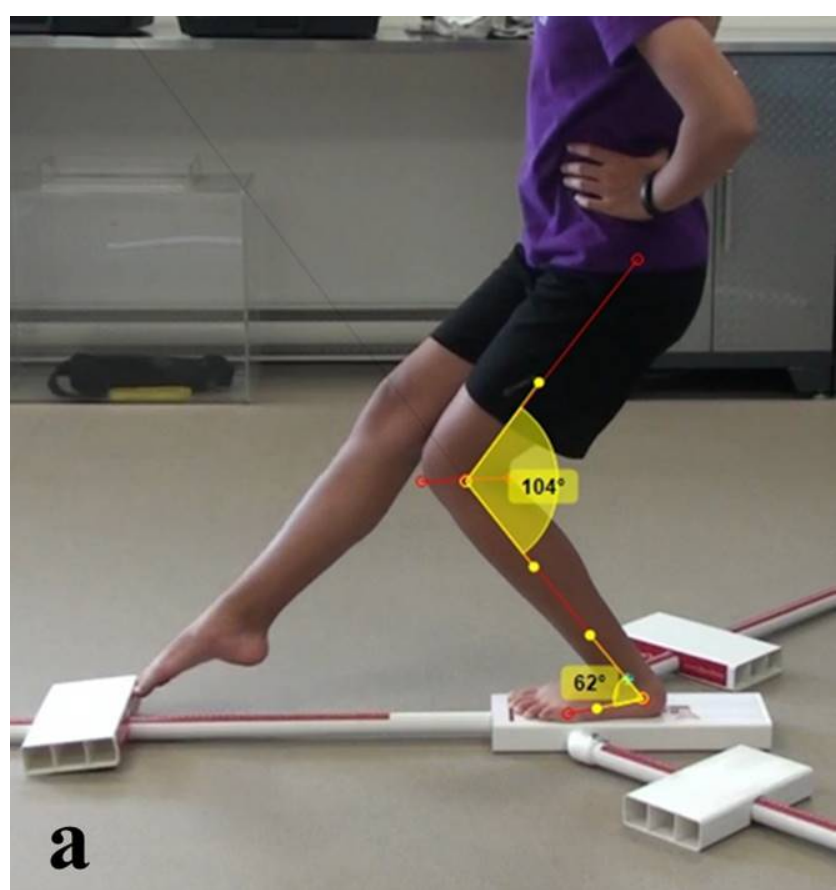

Figure 1a: The degree of knee joint flexion and ankle joint dorsiflexion in the sagittal plane.

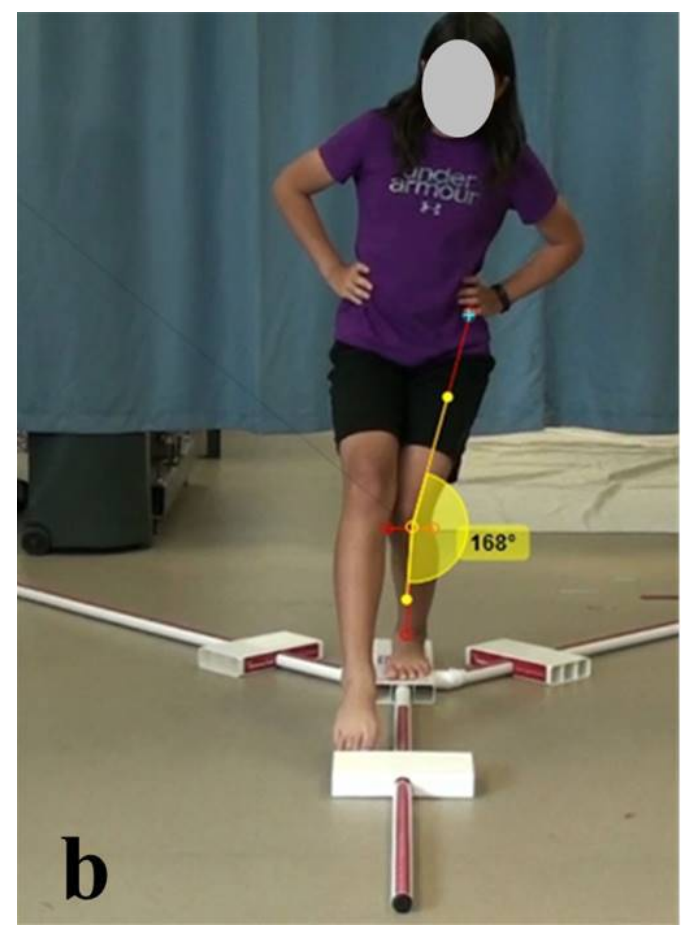

Figure 1b: The knee valgus angle in the frontal plane at the point of maximal ANT reach distance of the YBT.

\section{RESULTS}

Results indicated that the ACL-injured group was significantly older than then the healthy group (Healthy: $14.0 \pm 1.3$ yrs; ACL injured: $16.3 \pm 1.6$ yrs, $p<0.001)$. With the ex- 
Table 1: Y-Balance Test (YBT) reach distances for all participants [mean $\pm \mathrm{SD}$, (95\% CI)]

\begin{tabular}{|l|c|c|c|}
\hline \multicolumn{1}{|c|}{ YBT Reach Direction } & Healthy $(\mathrm{n}=\mathbf{2 5})$ & ACL-Injured $(\mathrm{n}=\mathbf{1 0})$ & $\mathrm{p}$-value \\
\hline & & $64.5 \pm 5.9$ & \\
Anterior (ANT) & $65.1 \pm 6.0$ & $(60.3-68.7)$ & 0.80 \\
(\%LL) & $(62.6-67.6)$ & $96.0 \pm 9.5$ & 0.30 \\
Posteromedial (PM) & $99.3 \pm 7.5$ & $(89.2-102.8)$ & \\
(\%LL) & $(96.2-102.4)$ & $95.1 \pm 8.4$ & 0.40 \\
Posterolateral (PL) & $97.7 \pm 9.0$ & $(89.1-101.1)$ & \\
(\%LL) & $(94.0-101.4)$ & & \\
\hline
\end{tabular}

\%LL - Percentage of limb length

Table 2: Lower extremity kinematic data for all participants when performing the YBT anterior reach movement $[$ mean \pm SD, (95\% CI)]

\begin{tabular}{|l|c|c|c|}
\hline & Healthy $(\mathbf{n}=\mathbf{2 5})$ & ACL-Injured (n=10) & p-value \\
\hline Knee flexion angle & & $70.9 \pm 9.2$ & \\
(degrees) & $66.4 \pm 10.9$ & $(64.3-77.5)$ & 0.25 \\
Ankle dorsiflexion angle & $(61.9-70.9)$ & $27.2 \pm 4.9$ & \\
(degrees) & $28.4 \pm 4.6$ & $(23.7-30.7)$ & 0.49 \\
Knee valgus angle & $(26.5-30.3)$ & $5.2 \pm 7.8$ & \\
(degrees) & $3.9 \pm 6.4$ & $(-0.4-10.9)$ & 0.61 \\
\hline
\end{tabular}

Table 3: Relationship between YBT anterior reach scores and lower extremity kinematic data

\begin{tabular}{|l|c|c|c|c|}
\hline \multirow{2}{*}{} & \multicolumn{2}{|c|}{ Healthy $(\mathrm{n}=\mathbf{2 5})$} & \multicolumn{2}{|c|}{ ACL-Injured ( $\mathrm{n}=10)$} \\
\cline { 2 - 5 } & $\mathrm{r}$ & $\mathrm{p}$-value & $\mathrm{r}$ & $\mathrm{p}$-value \\
\hline Knee flexion angle (degrees) & 0.37 & 0.07 & 0.43 & 0.21 \\
\hline Ankle dorsiflexion angle (degrees) & $0.42^{*}$ & 0.04 & 0.06 & 0.87 \\
\hline Knee valgus angle (degrees) & $-0.40^{*}$ & 0.05 & 0.30 & 0.52 \\
\hline
\end{tabular}

$* \mathrm{p} \leq 0.05$

ception of knee joint laxity (Healthy: $6.5 \pm 2.6 \mathrm{~mm}$; ACL-injured: $11.7 \pm 1.7 \mathrm{~mm}, \mathrm{p}<.001)$, there were no significant differences between the anthropometric measurements of the two groups. All participants had a healthy BMI (Healthy: $22.3 \pm 4.8 \mathrm{~kg} / \mathrm{m}^{2}$; ACL injured: $\left.24.8 \pm 4.6 \mathrm{~kg} / \mathrm{m}^{2}, \mathrm{p}=0.18\right)$, and were predominantly post-pubertal adolescents (Healthy: $68 \%$; ACL injured: $60 \%$ ) who participated in a variety of recreational sporting activities including basketball, volleyball, soccer, hockey, and dance. For ACL-injured participants, the mean time from injury to the baseline examination for this study was 143 days (range: 24-365).

YBT reach distances and lower extremity kinematic data for all subjects (organized by group) are presented in Tables 1 and 2 . There were no significant differences in YBT reach distances or lower extremity kinematic data when comparing healthy and ACL-injured groups.

Table 3 illustrates the relationship between the YBT ANT reach distance and lower extremity joint angles. Pearson correlation coefficient testing for the healthy subjects suggested that there were weak relationships $(0.25-0.49)$ between the YBT ANT reach distance and the degree of ankle dorsiflexion (positive), the degree of knee valgus (negative), and that there was a trend towards a positive relationship with the degree of knee flexion. These relationships were only significant for the ankle dorsiflexion and knee valgus angles. Analysis of data for the ACL-injured group suggested that there were no relationships between lower extremity joint angles and YBT ANT reach scores.

Using YBT ANT reach data, 95\% confidence intervals (CI) were calculated for each group. Subjects were then stratified into three groups: (1) Above 95\% CI $=72.5 \pm 2.8 \%$; (2) Within 95\% CI $=64.7 \pm 1.5 \%$; and (3) Below 95\% CI = 58.2 \pm $3.3 \%$. A comparison of the kinematic data across the three groups indicated that there were no significant differences for either the healthy participants or the ACL injured participants (Table 4).

These same three YBT ANT reach distance groupings (above, within or below 95\% CI) where then used to perform single-subject analyses. Data illustrated that there was little consistency in the movement patterns demonstrated about the ankle and knee joints by either the healthy or ACL-injured subjects when performing the ANT reach of the 
Table 4: Kinematic data (mean \pm SD) for healthy and ACL-injured participants categorized by YBT anterior reach distance

\begin{tabular}{|l|c|c|c|}
\hline \multicolumn{1}{|c|}{ Healthy } & $\begin{array}{c}\text { Above 95\% Cl } \\
(\mathbf{n = 7})\end{array}$ & $\begin{array}{c}\text { Within 95\% Cl } \\
(\mathbf{n = 1 1})\end{array}$ & $\begin{array}{c}\text { Below 95\% Cl } \\
(\mathbf{n}=7)\end{array}$ \\
\hline Knee flexion angle (degrees) & $70.3 \pm 15.0$ & $68.6 \pm 6.3$ & $58.9 \pm 9.6$ \\
Ankle dorsiflexion angle (degrees) & $30.0 \pm 3.5$ & $29.5 \pm 3.0$ & $25.1 \pm 6.4$ \\
Knee valgus angle (degrees) & $2.6 \pm 8.9$ & $3.5 \pm 6.1$ & $5.9 \pm 3.8$ \\
\hline \multicolumn{1}{|c|}{ ACL-injured } & $\begin{array}{c}\text { Above 95\% Cl } \\
(\mathbf{n}=2)\end{array}$ & $\begin{array}{c}\text { Within 95\% Cl } \\
(\mathbf{n}=4)\end{array}$ & $\begin{array}{c}\text { Below 95\% Cl } \\
(\mathbf{n}=4)\end{array}$ \\
\hline Knee flexion angle (degrees) & $81.0 \pm 8.5$ & $69.8 \pm 5.7$ & $67.0 \pm 10.2$ \\
Ankle dorsiflexion angle (degrees) & $32.0 \pm 1.4$ & $22.8 \pm 4.6$ & $29.3 \pm 2.1$ \\
Knee valgus angle (degrees) & $6.0 \pm 14.1$ & $5.5 \pm 8.5$ & $4.5 \pm 6.4$ \\
\hline
\end{tabular}

Table 5: Single subject categorical comparison of kinematic data and YBT anterior reach distance for healthy participants

\begin{tabular}{|c|c|c|c|}
\hline YBT & Knee flexion angle & Ankle dorsiflexion angle & Knee valgus angle \\
\hline \multirow{7}{*}{ Above $95 \% \mathrm{Cl}$} & + & 0 & - \\
\hline & + & + & 0 \\
\hline & + & 0 & + \\
\hline & - & 0 & - \\
\hline & + & 0 & - \\
\hline & 0 & + & + \\
\hline & - & + & - \\
\hline \multirow{11}{*}{ Within $95 \% \mathrm{Cl}$} & + & + & + \\
\hline & 0 & 0 & 0 \\
\hline & 0 & + & - \\
\hline & 0 & + & 0 \\
\hline & - & 0 & + \\
\hline & + & 0 & + \\
\hline & 0 & + & 0 \\
\hline & + & 0 & - \\
\hline & + & 0 & - \\
\hline & 0 & 0 & + \\
\hline & 0 & - & + \\
\hline \multirow{7}{*}{ Below 95\% Cl } & 0 & + & 0 \\
\hline & + & - & 0 \\
\hline & 0 & - & 0 \\
\hline & - & - & 0 \\
\hline & 0 & 0 & + \\
\hline & - & 0 & 0 \\
\hline & - & 0 & 0 \\
\hline
\end{tabular}

Legend: + above 95\% CI; 0 within 95\% CI; and - below 95\% CI

YBT (Tables $\underline{5} \& \underline{6})$.

\section{DISCUSSION}

This is one of the first investigations to examine lower ex- tremity kinematics during performance of the YBT in both at-risk healthy and ACL-injured adolescent females. Results suggested that there were no significant differences between the healthy and ACL-injured subjects on YBT scoring, or the lower extremity joint angles observed when partic- 
Table 6: Single subject categorical comparison of kinematic data and YBT anterior reach distance for ACLinjured participants.

\begin{tabular}{|c|c|c|c|}
\hline YBT & Knee flexion angle & Ankle dorsiflexion angle & Knee valgus angle \\
\hline \multirow{3}{*}{ Above 95\% Cl } & + & + & + \\
\hline \multirow{3}{*}{ Within 95\% Cl } & + & + & + \\
& + & - & + \\
& 0 & - & 0 \\
\hline \multirow{3}{*}{ Below 95\% Cl } & + & - & 0 \\
& - & + & + \\
\hline
\end{tabular}

Legend: + above 95\% CI; 0 within $95 \% \mathrm{CI}$; and - below $95 \% \mathrm{CI}$

ipants performed the YBT ANT reach direction. Data for the healthy adolescents indicated that there were only weak correlations between scoring on the YBT and the angle of ankle dorsiflexion (larger YBT scores were associated with a greater degree of ankle dorsi-flexion) and the angle of knee valgus (larger YBT scores were associated with less knee valgus), but there was little relationship between the angle of knee flexion and YBT ANT reach scoring. Results for the ACL-injured group suggested there were no relationships between any of the lower extremity kinematic measures and ANT reach scoring on the YBT. Interestingly, a comparison of subjects when grouped by their performance on the YBT ANT reach (above, within or below the 95\% CI) also failed to identify any consistencies between YBT scoring and lower extremity joint angles (for example: a girl who scored well on the YBT was just as likely to have a large knee flexion angle as a girl who performed poorly). A similar result was observed when single-subject analysis was performed. These findings serve to reinforce the notion that adolescent females frequently utilize a variety of lower extremity movement strategies when performing functional movement tasks typically observed in sport, and suggest that ANT reach scoring on the YBT may offer limited insight regarding lower extremity joint kinematics, and ACL-injury risk in a physically active adolescent female population.

This investigation is among the first to report both dynamic balance and kinematic data that is specific to both a healthy and ACL injured adolescent female population who participate in a variety of recreational sporting activities and is nearing or has recently reached physical maturation. While data indicated that the healthy group was much younger than the ACL injured group, the only significant difference observed in the anthropometry of the two groups was on the parameter of knee joint laxity (ACL deficient group > healthy group). As such, the results help to fill a significant void that currently exists within the biomechanical and neuromuscular testing literature, and provides important baseline data that is generalizable to a much larger population of adolescent females that is at significant risk for ACL injury during sports participation.
The results serve to extend the findings of previous investigations on dynamic balance which have focused on athletes from specific sports, ${ }^{6,20}$ older age groups, ${ }^{40-42}$ or competitive sport. ${ }^{22,43}$ The literature indicates that dynamic balance scoring can vary greatly, and can be influenced by a host of factors including testing methodologies, ${ }^{26,38}$ the precision of the value reported, ${ }^{40,44-48}$ and the study population's age, ${ }^{40-42}$ sex, 6,20,46,48,49 sport, 6,20 and competitive level. ${ }^{22,43,50}$ YBT scoring for our healthy adolescent female population fell within the range of expected scores based on participants age, gender and competitive level - scores were slightly higher than those previously reported for adults;26,27,38 slightly lower than adolescent athlete scoring reported for both sexes $; 33,51$ and most similar to scoring from older female participants. 52,53 Normal function of the proprioceptive, vestibular, and visual systems are all important for dynamic balance, and each of these systems are still maturing and developing during the adolescent period. ${ }^{44,54-56}$ As such, it is possible that gender and biological changes associated with aging and physical maturation had a significant effect on YBT scoring in the present study, ${ }^{53,57}$ and caution should be used when comparing these data with previous reports for an adult population.

Kinematic data from the current investigation provides important information about lower extremity joint movements during the execution of the YBT ANT reach by a population of healthy adolescent females at significant risk for lower extremity injury. Sagittal plane data for the angle of ankle dorsiflexion ${ }^{25-27}$ and the angle of knee flexion $^{23,25-27,58}$ were comparable to previous reports involving dynamic balance testing. Within a general population, dorsiflexion angles typically range from $32^{\circ}$ to $39^{\circ}$, and knee flexion angles have been reported to range from $51^{\circ}$ to $68^{\circ} .23,25-27,58$ To date, only one previous investigation has examined the knee valgus angle (mean $=14.15^{\circ} \pm 8.36$ ) during performance of the YBT, and this study involved a physically mature population of co-ed university students (mean age of $22.7 \pm 2.2$ years). ${ }^{27}$ Participant knee valgus angle for our adolescent female participants were significantly lower 
than values reported in this earlier study, and are much closer to values reported in another study which evaluated a single leg squat task in 30 physically active young females. ${ }^{9}$

This investigation also examined YBT ANT reach distance and lower extremity kinematics in an exploratory group of ACL-injured participants. Data indicated that YBT reach distance and joint angles were not significantly different when comparing healthy and ACL-injured adolescent females. These findings may suggest that our ACL-injured subjects were able to use some form of neuromuscular control in order to compensate for their ligamentous instability in their knee. Previous research does suggest that a percentage of ACL injured patients, often referred to as "copers", are capable of coordinating muscle activity to dynamically stabilize the knee and resume preinjury activities without episodes of the knee giving-way. ${ }^{59}$ Having said this, the current investigation of the YBT is the first to include ACLinjured adolescent individuals. Previous investigations of clinical dynamic balance using subjects with ACL injury have used less reliable dynamic balance testing protocols such as the Star Excursion Balance Test (SEBT). ${ }^{8,33}$ These studies found differences between the healthy controls and the ACL injured participants for the anterior reach direction. ${ }^{8}$ Although the YBT and SEBT testing protocols are similar, research suggests that the anterior reach distance performance and kinematic profiles of test subjects are different, and that each test imposes different neuromuscular demands and postural-control strategies on the participant. ${ }^{26,38,60}$ Thus caution should be used when comparing results from the different forms of dynamic balance testing, and further neuromuscular research which targets ACL-injured subjects is required in order to clarify inconsistencies, and determine whether a difference really exists between injured and healthy limbs during execution of the YBT. 8,33

Research on an adult population has previously indicated that the angle of ankle dorsiflexion is the best single kinematic predictor of YBT scoring in the anterior reach direction. ${ }^{27,61}$ Anterior reach YBT scores have also been reported to have a moderate correlation with the angle of knee flexion. But, no significant relationship has been established between YBT scoring and the angle of knee valgus. ${ }^{27} \mathrm{Re}$ sults for a healthy adolescent female population were in agreement with the results of these earlier investigations, and suggested that a positive weak correlation existed between YBT scoring and the angle of ankle dorsiflexion (ie. subjects who achieved greater ankle dorsiflexion tended to be able reach further on the YBT). However, in contrast to these earlier reports data from this investigation suggested that there was no correlation between the angle of knee flexion and YBT anterior reach scoring, and that a negative correlation existed between a subject's knee valgus angle and their YBT score (ie. as the angle of knee valgus increased, a subjects YBT anterior reach distance actually decreased). Previous research specific to an adolescent female population has suggested that kinematic assessment of the knee joint may be more important than the ankle joint as females demonstrate a larger dynamic knee valgus position than males when performing functional tasks such as jump landing and single leg squats. ${ }^{62-64}$ Dynamic knee valgus measures during the execution of functional tasks (such as the YBT) are reported to be predictive of risk for
ACL injury in adolescent females. ${ }^{4,5,7,12}$ Results from this investigation are in agreement with other reports which suggest that lower YBT scores ${ }^{19-22}$ and greater knee valgus angles $4,5,7,12$ are frequently observed in populations who are at increased risk of ACL injury.

In contrast to the healthy subjects, data for the ACLinjured participants suggested that there was no relationship between lower extremity joint angles and YBT anterior reach scoring. While the large range in scoring and small sample size may have contributed to this finding, it is also possible that anterior/posterior joint instability and participant movement apprehension associated with ACL deficiency resulted in participants being more hesitant or guarded when performing the YBT anterior reach movement. ${ }^{65}$ As a result, the ACL injured subjects may have subconsciously utilized movement strategies the involved greater range of motion at the hip joint or within the trunk region to achieve similar reach distances while minimizing movements about their injured knee. ${ }^{23}$

Finally, a comparison of the kinematic data between the healthy and ACL-injured groups suggested that there were no significant differences in ankle and knee joint angles when performing the YBT ANT reach direction. These results were confirmed when data was organized by YBT confidence intervals and by single-subject analysis. The sensorimotor system that regulates balance and postural awareness relies on information from the visual, vestibular and somatosensory subsystems. ${ }^{66}$ However, pubertal growth is reported to inhibit the sensorimotor functions of the lower extremity and lead to awkward movement patterns. ${ }^{56}$ The variability of movement strategies we observed may be attributed to the fact that our participants were still progressing through or recently completed the maturation process, and as such their neuromuscular control and intersegmental limb coordination were still developing. ${ }^{56}$ During execution of the YBT, the only instructions given to subjects were to push the reach indicator as far as possible along the pipe in the reach direction while maintaining a unilateral stance with their hands on hips. No tips on how to enhance performance were given. ${ }^{16,46}$ Kinematic data for individual subjects would seem to suggest that adolescent females use a variety of movement strategies to achieve maximal reach distance, including ankle dorsiflexion, knee flexion and knee valgus. Standardized placement of the hands on hips was used to minimize the influence of upper body/extremity sway, although variations in hip and trunk movement were unavoidable. Balance-correcting strategies of the trunk and hip are often used to maintain one's center of mass over a base of support and prevent loss of balance during a lower limb reach. ${ }^{23,27}$ Other investigations indicate that a similar anterior reach distance can be achieved by either flexing the hip and knee of the stance limb, or creating a Trendelenburg position, adducting the hip of the stance limb to lengthen the reach limb. ${ }^{23}$ Lack of stability at the trunk and hip is suggested to contribute to lateral trunk and Trendelenburg positions, and subsequently a knee valgus position. ${ }^{4,5,7}$ Unfortunately in this study, hip and trunk kinematic data were unable to be collected because the video cameras were positioned to collect information exclusive to the lower extremity. The specific influence of trunk and pelvic positions on the kinematics of 
the lower extremity may reveal more information about the role of distinct or varied positional stabilization strategies during execution of the YBT, and should be examined in future investigations.

This study is not without limitation. The use of 2D video analysis restricted kinematic analysis to only the anterior reach direction of the YBT. Kinematic analysis of posterolateral \& posteromedial reach directions are much more complex (due to the additional rotational movements of the trunk and lower extremity), and thus required more sophisticated 3D motion-capture equipment that was beyond the scope of this investigation. ${ }^{26,67}$ Additionally, due to time and lab space constraints in our data collection protocol (eg. multiple participants were being simultaneously evaluated) videotaping was restricted to only the first test trial for each subject. As such, it is possible that subjects may have altered their kinematic approach to performing the YBT over the three consecutive trials of the testing methodology. Kinematic analysis of each of these individual trials may have helped to provide a more reliable depiction of the relationship between lower extremity joint position and scoring on the YBT.

\section{CONCLUSION}

The results of this investigation indicate that there were no significant differences in YBT scoring and lower extremity kinematic data in the anterior reach direction when comparing the movement patterns of healthy and ACL-injured adolescent females. Additionally, both groups utilized a variety of lower extremity movement strategies when performing the functional movement task. Reach scoring on the YBT may offer limited insight regarding lower extremity joint kinematics, and ACL-injury risk in a physically active adolescent female population. Although beyond the scope of this study, results from future prospective, longitudinal investigations of both healthy and ACL-injured adolescent females could be used to identify the physical and neurological parameters that influence knee joint motion in this at-risk sporting population.

\section{CONFLICTS OF INTEREST}

The authors declare that there is no conflict of interest.

Submitted: May 29, 2020 CDT, Accepted: November 08, 2020

CDT 


\section{REFERENCES}

1. Sanders TL, Maradit Kremers H, Bryan AJ, et al. Incidence of anterior cruciate ligament tears and reconstruction: A 21-year population-based study. Am J Sports Med. 2016;44(6):1502-1507. doi:10.1177/0 $\underline{363546516629944}$

2. Stanley LE, Kerr ZY, Dompier TP, Padua DA. Sex differences in the incidence of anterior cruciate ligament, medial collateral ligament, and meniscal injuries in collegiate and high school sports: 2009-2010 through 2013-2014. Am J Sports Med. 2016;44(6):1565-1572. doi:10.1177/036354651663092 7

3. Gornitzky AL, Lott A, Yellin JL, Fabricant PD, Lawrence JT, Ganley TJ. Sport-specific yearly risk and incidence of anterior cruciate ligament tears in high school athletes: A systematic review and metaanalysis. Am J Sports Med. 2016;44(10):2716-2723. do i: $10.1177 / 0363546515617742$

4. Myer GD, Brent JL, Ford KR, Hewett TE. Real-time assessment and neuromuscular training feedback techniques to prevent ACL injury in female athletes. Strength Cond J. 2011;33(3):21-35. doi:10.1519/SSC.0b 013e318213afa8

5. Hewett TE, Myer GD, Ford KR, et al. Biomechanical measures of neuromuscular control and valgus loading of the knee predict anterior cruciate ligament injury risk in female athletes: A prospective study. Am J Sports Med. 2005;33(4):492-501. doi:10.1177/036354 $\underline{6504269591}$

6. Filipa A, Byrnes R, Paterno MV, Myer GD, Hewett TE. Neuromuscular training improves performance on the star excursion balance test in young female athletes. J Orthop Sports Phys Ther. 2010;40(9):551-558. doi:10.2519/jospt.2010.3325

7. Paterno MV, Schmitt LC, Ford KR, et al. Biomechanical measures during landing and postural stability predict second anterior cruciate ligament injury after anterior cruciate ligament reconstruction and return to sport. Am J Sports Med. 2010;38(10):1968-1978. doi:10.1177/03635465103760 $\underline{53}$

8. Herrington L, Hatcher J, Hatcher A, McNicholas M. A comparison of Star Excursion Balance Test reach distances between ACL deficient patients and asymptomatic controls. The Knee. 2009;16(2):149-152. doi:10.1016/j.knee.2008.10.004

9. Herrington L. Knee valgus angle during single leg squat and landing in patellofemoral pain patients and controls. The Knee. 2014;21(2):514-517. doi:10.1016/ j.knee.2013.11.011
10. Räisänen A, Pasanen K, Krosshaug T, Avela J, Perttunen J, Parkkari J. Single-leg squat as a tool to evaluate young athletes' frontal plane knee control. Clin J Sport Med. 2016;26(6):478-482.

11. Munro A, Herrington L, Carolan M. Reliability of 2-dimensional video assessment of frontal-plane dynamic knee valgus during common athletic screening tasks. J Sport Rehabil. 2012;21(1):7-11.

12. Ugalde V, Brockman C, Bailowitz Z, Pollard CD. Single leg squat test and its relationship to dynamic knee valgus and injury risk screening. $P M \& R$. 2015;7(3):229-235. doi:10.1016/i.pmri.2014.08.361

13. Sigward SM, Ota S, Powers CM. Predictors of frontal plane knee excursion during a drop land in young female soccer players. J Orthop Sports Phys Ther. 2008;38(11):661-667. doi:10.2519/jospt.2008.26 $\underline{95}$

14. Rabin A, Portnoy S, Kozol Z. The association of ankle dorsiflexion range of motion with hip and knee kinematics during the lateral step-down test. J Orthop Sports Phys Ther. 2016;46(11):1002-1009. doi:10.251 9/jospt.2016.6621

15. Paz G, Maia M, Farias D, et al. Kinematic analysis of knee valgus during drop vertical jump and forward step-up in young basketball players. Int J Sports Phys Ther. 11(2):212-219.

16. Plisky PJ, Gorman PP, Butler RJ, Kiesel KB, Underwood FB, Elkins B. The reliability of an instrumented device for measuring components of the star excursion balance test. North Am J Sports Phys Ther. 2009;4(2):92-99.

17. Shaffer SW, Teyhen DS, Lorenson CL, et al. Ybalance test: a reliability study involving multiple raters. Mil Med. 2013;178(11):1264-1270. doi:10.720 5/MILMED-D-13-00222

18. Gribble PA, Hertel J, Plisky P. Using the star excursion balance test to assess dynamic posturalcontrol deficits and outcomes in lower extremity injury: a literature and systematic review. J Athl Train. 2012;47(3):339-357.

19. Gribble PA, Terada M, Beard MQ, et al. Prediction of lateral ankle sprains in football players based on clinical tests and body mass index. Am J Sports Med. 2016;44(2):460-467. doi:10.1177/0363546515614585 
20. Plisky PJ, Rauh MJ, Kaminski TW, Underwood FB. Star Excursion Balance Test as a predictor of lower extremity injury in high school basketball players. I Orthop Sports Phys Ther. 2006;36(12):911-919.

21. Butler RJ, Lehr ME, Fink ML, Kiesel KB, Plisky PJ. Dynamic balance performance and noncontact lower extremity injury in college football players: An initial study. Sports Health Multidiscip Approach.

2013;5(5):417-422. doi:10.1177/1941738113498703

22. Smith CA, Chimera NJ, Warren M. Association of $\mathrm{Y}$ balance test reach asymmetry and injury in division I athletes. Med Sci Sports Exerc. 2015;47(1):136-141. $\underline{\mathrm{d}}$ oi:10.1249/MSS.0000000000000380

23. Delahunt E, Chawke M, Kelleher J, et al. Lower limb kinematics and dynamic postural stability in anterior cruciate ligament-reconstructed female athletes. J Athl Train. 2013;48(2):172-185. doi:10.408 5/1062-6050-48.2.05

24. Fitzgerald D, Trakarnratanakul N, Smyth B, Caulfield B. Effects of a wobble board-based therapeutic exergaming system for balance training on dynamic postural stability and intrinsic motivation levels. J Orthop Sports Phys Ther. 2010;40(1):11-19. doi:10.2519/jospt.2010.3121

25. Eltoukhy M, Kuenze C, Oh J, Wooten S, Signorile J. Kinect-based assessment of lower limb kinematics and dynamic postural control during the star excursion balance test. Gait Posture. 2017;58:421-427. doi:10.1016/j.gaitpost.2017.09.010

26. Fullam K, Caulfield B, Coughlan GF, Delahunt E. Kinematic analysis of selected reach directions of the star excursion balance test Compared with the Ybalance test. J Sport Rehabil. 2014;23(1):27-35. doi:1 0.1123/JSR.2012-0114

27. Kang M-H, Kim G-M, Kwon O-Y, Weon J-H, Oh J-S, An D-H. Relationship between the kinematics of the trunk and lower extremity and performance on the Ybalance test. $P M \& R$. 2015;7(11):1152-1158. doi:10.10 16/j.pmrj.2015.05.004

28. Fitzgerald GK, Axe MJ, Snyder-Mackler L. Proposed practice guidelines for non-operative anterior cruciate ligament rehabilitation of physically active individuals. J Orthop Sports Phys Ther. 2000;30(4):194-203.

29. Daniel DM, Stone ML, Dobson BE, Fithian DC, Rossman DJ, Kaufman KR. Fate of the ACL-injured patient: A prospective outcome study. Am J Sports Med. 1994;22(5):632-644. doi:10.1177/036354659402 $\underline{200511}$
30. Davies PL, Rose JD. Motor skills of typically developing adolescents: awkwardness or improvement? Phys Occup Ther Pediatr. 2000;20(1):19-42.

31. Gribble PA, Kelly SE, Refshauge KM, Hiller CE. Interrater reliability of the star excursion balance test. J Athl Train. 2013;48(5):621-626. doi:10.4085/10 62-6050-48.3.03

32. Gribble PA, Hertel J. Considerations for normalizing measures of the star excursion balance test. Meas Phys Educ Exerc Sci. 2003;7(2):89-100. doi:1 0.1207/S15327841MPEE0702 3

33. Zult T, Gokeler A, van Raay JJAM, Brouwer RW, Zijdewind I, Hortobágyi T. An anterior cruciate ligament injury does not affect the neuromuscular function of the non-injured leg except for dynamic balance and voluntary quadriceps activation. Knee Surg Sports Traumatol Arthrosc. 2017;25(1):177-183. d oi:10.1007/s00167-016-4335-3

34. Dill KE, Begalle R, Frank B, Zinder S, Padua DA. Altered knee and ankle kinematics during squatting in those with limited weight-bearing lunge ankledorsiflexion range of motion. J Athl Train. 2014;49(6):723-732. doi:10.4085/1062-6050-49.3.29

35. Piva SR, Fitzgerald K, Irrgang JJ, et al. Reliability of measures of impairments associated with patellofemoral pain syndrome. BMC Musculoskelet Disord. 2006;7(1):33.

36. Hamilton N, Weimar W, Luttgens K. Kinesiology: Scientific Basis of Human Motion. 12 edition. New York, NY: McGraw-Hill Education; 2011.

37. Bland JM, Altman D. Statistical methods for assessing agreement between two methods of clinical measurement. The lancet. 1986;327(8476):307-310.

38. Coughlan GF, Fullam K, Delahunt E, Gissane C, Caulfield BM. A comparison between performance on selected directions of the star excursion balance test and the Y balance test. J Athl Train. 2012;47(4):366.

39. Portney LG, Watkins MP. Foundations of Clinical Research: Applications to Practice. 3rd ed. Pearson Education Inc; 2009.

40. Hudson C, Garrison JC, Pollard K. Y-balance normative data for female collegiate volleyball players. Phys Ther Sport. 2016;22:61-65. doi:10.1016/ j.ptsp.2016.05.009

41. Munro AG, Herrington LC. Between-session reliability of the star excursion balance test. Phys Ther Sport. 2010;11(4):128-132. doi:10.1016/j.ptsp.2010.0 7.002 
42. van Lieshout R, Reijneveld EA, van den Berg SM, et al. Reproducibility of the modified sart excursion balance test composite and specific reach direction scores. Int J Sports Phys Ther. 2016;11(3):356.

43. Butler RJ, Southers C, Gorman PP, Kiesel KB, Plisky PJ. Differences in soccer players' dynamic balance across levels of competition. J Athl Train. 2012;47(6):616-620. doi:10.4085/1062-6050-47.5.14

44. Breen EO, Howell DR, Stracciolini A, Dawkins C, Meehan W. Examination of age-related differences on clinical tests of postural stability. Sports Health. 2016;8(3):244-249.

45. Chimera NJ, Smith CA, Warren M. Injury history, sex, and performance on the functional movement screen and Y balance test. J Athl Train. 2015;50(5):475-485. doi:10.4085/1062-6050-49.6.02

46. Faigenbaum AD, Myer GD, Fernandez IP, et al. Feasibility and reliability of dynamic postural control measures in children in first through fifth grades. Int $J$ Sports Phys Ther. 2014;9(2):140-148.

47. Holden S, Boreham C, Doherty C, Wang D, Delahunt E. Dynamic postural stability in young adolescent male and female athletes. Pediatr Phys Ther. 2014;26(4):447-452. doi:10.1097/PEP.000000000 $\underline{0000071}$

48. Holden S, Boreham C, Doherty C, Wang D, Delahunt E. A longitudinal investigation into the progression of dynamic postural stability performance in adolescents. Gait Posture. 2016;48:171-176. doi:10.1016/i.gaitpost.2016.04.019

49. Brophy RH, Staples JR, Motley J, Blalock R, StegerMay K, Halstead M. Young females exhibit decreased coronal plane postural stability compared to young males. HSS J. 2016;12(1):26-31. doi:10.1007/s11420-0 15-9458-4

50. Engquist KD, Smith CA, Chimera NJ, Warren M. Performance comparison of student-athletes and general college students on the functional movement screen and the Y balance test. J Strength Cond Res. 2015;29(8):2296-2303.

51. Gorman PP, Butler RJ, Rauh MJ, Kiesel K, Plisky PJ. Differences in dynamic balance scores in one sport versus multiple sport high school athletes. Int J Sports Phys Ther. 2012;7(2):148-153.

52. Ambegaonkar J, Mettinger L, Caswell S, Burtt A, Cortes N. Relationships between core endurance, hip strength, and balance in collegiate female athletes. Int I Sports Phys Ther. 2014;9(5):604-616.
53. Lee D-K, Kang M-H, Lee T-S, Oh J-S. Relationships among the $\mathrm{Y}$ balance test, Berg Balance Scale, and lower limb strength in middle-aged and older females. Braz J Phys Ther. 2015;19(3):227-234. d oi:10.1590/bjpt-rbf.2014.0096

54. Steindl R, Kunz K, Schrott-Fischer A, Scholtz A. Effect of age and sex on maturation of sensory systems and balance control. Dev Med Child Neurol. 2006;48(06):477. doi:10.1017/S0012162206001022

55. Lee H-M, Cheng C-K, Liau J-J. Correlation between proprioception, muscle strength, knee laxity, and dynamic standing balance in patients with chronic anterior cruciate ligament deficiency. The Knee. 2009;16(5):387-391. doi:10.1016/j.knee.2009.0 $\underline{1.006}$

56. Quatman-Yates CC, Quatman CE, Meszaros AJ, Paterno MV, Hewett TE. A systematic review of sensorimotor function during adolescence: a developmental stage of increased motor awkwardness? Br J Sports Med. 2012;46(9):649-655. d oi:10.1136/bjsm.2010.079616

57. Muehlbauer T, Gollhofer A, Granacher U. Associations between measures of balance and lowerextremity muscle strength/power in healthy individuals across the lifespan: A systematic review and meta-analysis. Sports Med. 2015;45(12):1671-1692. doi:10.1007/s40279-015-039 $\underline{0-\mathrm{Z}}$

58. Robinson R, Gribble P. Kinematic predictors of performance on the Star Excursion Balance Test. J Sport Rehabil. 2008;17(4):347-357.

59. Kaplan Y. Identifying individuals with an anterior cruciate ligament-deficient knee as copers and noncopers: A narrative literature review. J Orthop Sports Phys Ther. 2011;41(10):758-766. doi:10.2519/jo spt.2011.3384

60. Bulow A, Anderson JE, Leiter JR, MacDonald PB, Peeler J. The modified star excursion balance and Ybalance test results differ when assessing physically active healthy adolescent females. Int J Sports Phys Ther. 2019;14(2):192-203.

61. Hoch MC, Staton GS, McKeon PO. Dorsiflexion range of motion significantly influences dynamic balance. J Sci Med Sport. 2011;14(1):90-92. doi:10.101 6/j.jsams.2010.08.001

62. Ford KR, Myer GD, Hewett TE. Longitudinal effects of maturation on lower extremity joint stiffness in adolescent athletes. Am J Sports Med. 2010;38(9):1829-1837. doi:10.1177/036354651036742 $\underline{5}$ 
63. Ford KR, Myer GD, Hewett TE. Valgus knee motion during landing in high school female and male basketball players. Med Sci Sports Exerc.

2003;35(10):1745-1750. doi:10.1249/01.MSS.0000089 346.85744.D9

64. Ford KR, Myer GD, Smith RL, Vianello RM, Seiwert SL, Hewett TE. A comparison of dynamic coronal plane excursion between matched male and female athletes when performing single leg landings. Clin Biomech. 2006;21(1):33-40. doi:10.1016/i.clinbiomec h.2005.08.010
65. Shimokochi Y, Shultz SJ. Mechanisms of noncontact anterior cruciate ligament injury. J Athl Train. 2008;43(4):396-408.

66. Riemann BL, Lephart SM. The sensorimotor system, part II: the role of proprioception in motor control and functional joint stability. J Athl Train. 2002;37(1):80.

67. Ness B, Taylor A, Haberl M, Reuteman P, Borgert A. Clinical observation and analysis of movement quality during performance on the star excursion balance test. Int J Sports Phys Ther. 2015;10(2):168-176. 